\title{
Revisiting pulmonary rehabilitation during COVID-19 pandemic: a narrative review
}

\author{
Snehil Dixit ${ }^{1, *}$, Audrey Borghi-Silva ${ }^{2}$, Kalyana Chakravarthy Bairapareddy $^{3}$ \\ ${ }^{1}$ Department of Medical Rehabilitation Sciences, College of Applied Medical Sciences, King Khalid University, 61421 Abha, Kingdom of Saudi Arabia \\ ${ }^{2}$ Cardiopulmonary Physiotherapy Laboratory, Physiotherapy Department, Federal University of Sao Carlos, 13565-905 Sao Carlos, Brazil \\ ${ }^{3}$ College of Health sciences, University of Sharjah, 27272 Sharjah, United Arab Emirates \\ *Correspondence: snehildixit83@gmail.com (Snehil Dixit)
}

DOI:10.31083/j.rcm2202039

This is an open access article under the CC BY 4.0 license (https://creativecommons.org/licenses/by/4.0/).

Submitted: 1 February 2021 Revised: 18 May 2021 Accepted: 19 May 2021 Published: 30 June 2021

There has been an apparent association between the risks of complications with severe acute respiratory syndrome coronavirus 2 (SARS$\mathrm{CoV}-2)$ infection in patients with a history of existing chronic respiratory diseases during the pandemic of coronavirus disease 2019 (COVID-19). SARS-CoV-2 poses a severe risk in cardiopulmonary management. Moreover, chronic respiratory diseases may further amplify the risk of morbidity and mortality among the afflicted population in the pandemic era. The present review outlines the importance of pulmonary rehabilitation (PR) in persons with chronic respiratory diseases (Chronic obstructive pulmonary disease (COPD) and Asthma) during the COVID-19 era. In this context, amongst the population with a pre-existing pulmonary diagnosis who have contracted SARS-CoV-2, following initial medical management and acute recovery, exercise-based pulmonary rehabilitation (PR) may play a crucial role in long-term management and recovery. The energy conservation techniques will play a pragmatic role in PR of mild to moderate severity cases to counter post-COVID-19 fatigue. Moreover, there is also an urgent need to effectively address post-COVID-19 anxiety and depression, affecting the PR delivery system.

Keywords

COVID; COPD; Asthma; Exercise; Pulmonary rehabilitation

\section{Introduction}

The global emergence of the severe acute respiratory syndrome coronavirus $2(S A R S-C o V-2)$ was recognized as a novel and potentially life-threatening virus throughout the world. The emergence of this virus has led to coronavirus disease 2019 (COVID-19), which is triggered by a novel strain of coronavirus rapidly uses Angiotensin-Converting Enzyme 2 (ACE-2), which is identified to be the main receptor at the cellular level of the human airway system to gain access into the human host system and cause infection [1]. Of particular note, expression of ACE-2 is found to be increased in the lower respiratory tract of patients diagnosed with chronic obstructive pulmonary disease (COPD) [1] and Asthma.

Impaired host immunity, inflammatory responses, structural damages within the lung tissue, and increased mucus production are some of the pathophysiological changes in COPD, which may predispose factors for increased risk of clinical complications and poorer outcomes of SARS-CoV-2 infection [1]. The risk of increased severity of viral infection during the COVID-19 pandemic was found to be five times higher in patients with COPD [2]. It has also been observed that the COVID-19 virus usually spreads at surprising rates leading to a public health crisis with malfunctioning of the health care delivery system [3].

The systemic clinical manifestations of COVID-19 infection are fever, fatigue, myalgia, and arthralgia [4]. The lung inflammation storm caused due to SARS coronavirus 2 infection may progress to Acute Respiratory Distress Syndrome (ARDS) and may present with symptoms that may vary from mild to moderate cough, breathlessness, sputum production, and hemoptysis. The severe sign and symptoms are linked with cytokine storms, particularly interleukin-6 (IL-6), leading to a significant risk of death [5].

The severity of asthma attacks is highly linked to all-cause mortality. The chances of mortality were even higher with age, comorbidities, chronic smoking, male gender, and previous severe attack history [6]. It can also be postulated that the risk of mortality will be even higher in the current pandemic situation. Similarly, patients with pre-existing COPD conditions who come in contact with COVID-19 disease displays a greater chance of entrance in the intensive care unit with mechanical ventilation and poorer health-related outcomes [7].

According to Global Initiative for Obstructive Lung Disease (GOLD), patients with a known history of Chronic Obstructive Pulmonary Disease (COPD) are amongst the affected by COVID-19 infection [8]. The diagnosed cases of COPD across all stages of the disease carry an increased risk of COVID-19 infection, which may lead to severe lung complications in survivors [9]. Although the prevalence of COVID19 infection was found to be low, the severity of disease and mortality rates were found to be significantly high in diagnosed cases of COPD [10]. A proper management plan is essential for survivors of COVID-19 infection, and there is an emerging need for therapeutic strategies for patients with chronic lung diseases, including COPD $[11,12]$. 
There is significant existing evidence that COVID-19 infection leads to permanent lung scarring and damage [1214]. The symptoms related to COVID-19 infection were more severe in patients with pre-existing chronic respiratory conditions like COPD and only moderately affected Asthma [15]. It's been systematically established in clinical trials that physical training induces a reduction in proinflammatory cytokines' plasma levels [16].

The exercise-based Pulmonary rehabilitation (PR) program plays an imperative role during the recovery period in COVID survivors with a history of COPD or Asthma [17]. PR program goals are the restoration of physical, psychological, and social functions to their optimal level. These goals may well be considered for COVID-19, COPD/asthma survivors, given the possibilities of irreversible lung damage and long-term disabilities. Other vital problems related to fatigue will also need to be explored. Population suffering from moderate to severe symptoms of COVID-19 may experience chronic fatigue syndrome [18]. Thus, energy conservation techniques will be of vital importance for patients to adhere to the PR. Hence the primary objective of the current review is to explore the possible role of the PR program in the COVID-19 era for patients suffering from respiratory conditions of COPD and Asthma.

\section{Long term complications of COVID-19 and need for comprehensive long-term rehabilitation}

The chances for a COPD individual are 4-5 times higher to contact COVID-19 [19].

The patients with pre-existing lung diseases present with hemoptysis, chest pain, and exertional breathlessness [17]. The severity of breathlessness depends on the stage and chronicity of COPD and the extent of damage due to the COVID pandemic [20]. Acute respiratory distress syndrome (ARDS) is the most typical complication seen, with a reduction in lung compliance and ground-glass appearance of the lungs [20].

Apart from this, the most commonly observed symptoms of fatigue in COPD often go unnoticed. There is a need to emphasize fatigue-related problems in COPD for patients encountering fatigue-related symptoms in the recovery phase. Fatigue is usually described as a feeling of tiredness or being drained out of energy with little or no activity [21]. It is often associated with depression, frustration, and concentration problems [21]. Exacerbation of COPD problem with COVID-19 may further amplify the situation, prolonging the recovery phase.

\section{Pulmonary rehabilitation (PR): access to the rehabilitation services, therapeutic strategies, and beneficial effects \\ 3.1 Access to the rehabilitation services}

Respiratory diseases remain the most common cause of death worldwide [22]. Pulmonary rehabilitation is a system- atic treatment platform that focuses on patient-centered therapies for COPD, which includes education, self-management and exercise training with the objective to improve adherence of patients to healthy behaviourrs [23].

Access to PR program in rural areas is quite a challenge as frequent travel to these programs lowers patients' attendance. The identified barriers in accessing PR may include low referral rates and health system inefficiency [22]. By employing the home-based treatments, many of the patientrelated barriers, including regular turnout and adherence to the program, may be improved. Moreover, the fear of contracting coronavirus may keep many away from hospitals, even in cases of acute exacerbations [22]. Other barriers to COPD care that existed before COVID-19 continue to exist. For instance, the hospitals' wait times become much longer, communication and relationships with healthcare providers become much less efficient due to time constraints, and the stress from the pandemic may result in less adherence to selfmanagement measures.

As the prevalence of COPD increases with age in the COVID-19 era, the number of seniors who will require virtual COPD care will be higher than the younger generations. The elderly population commonly has a more challenging time navigating new technologies and may not be as open to virtual care as the younger generations [24].

The solution to easy access to rehabilitation services could be place-based (Step-down unit) and person-oriented strategies for health promotion to build local capacity in communities. It is also essential to use innovative technologies to supplement and enhance service delivery [25].

\subsection{Therapeutic strategies: components of exercise-based pulmonary rehabilitation}

Exercise rehabilitation is the keystone in the management of COPD in a pulmonary rehabilitation program. Exercise training can improve exercise performance in Asthma and COPD population [23].

\subsubsection{Education}

This is an imperative concept to the rehabilitation program. Education of the patient to non-pharmacological therapies in PR helps in improving adherence [26]. Education has a strong association with modification in health behaviors, self-management, and health-related outcomes [26]. The approach has been evolving, but the most commonly used methods are didactic lectures through web-based applications or social media platforms [23]. Knowledge about the disease process can empower the patients to understand disease pathophysiology, thereby resulting in ineffective interventions to manage the symptoms. In some research, it has been stated that education can play vital support for exercise training programs, thereby lowering the drop-outs rate in the PR. Hence education is a multifactorial aspect of the PR program. It must not be delivered in short courses but rather as an integral aspect to motivate the patients to continue the PR [23]. 
Another growing concern during the pandemic era for COPD patients is anxiety and depression or psychological issues. The critically ill patients surviving a protracted period of mechanical ventilation, complications, and long-drawnout disease process are profoundly debilitated and may require psychoeducation aspect of PR [27]. The psychoeducation aspect of the PR refers to dealing with the psychological issues that the patient encounters in the disease process of isolation and, in some instances, social stigma because of disease. Motivational interviews, mindfulness practice, education regarding the disease process, and breathing exercises can be highly beneficial in the road to recovery [27]. Psychological, educational support is currently recommended during the PR's educational component as the patient learns how to manage the signs and symptoms of anxiety and depression, which are usually associated with COPD and Asthma [28]. This concept has, however shown promising results in cardiac rehabilitation $[26,29]$.

\subsubsection{Exercise training}

Exercise training is the foundation stone of the PR program and helps in improving exercise performance and quality of living in Asthmatic and COPD patients [23, 30, 31]. Before starting any type of exercise training, it's vital to determine the participants' exercise capacity in the program. Exercise capacity evaluation can be done through cardiopulmonary exercise testing and field tests like six min walk test, shuttle walk test, and so on [32]. The rationale behind the exercise program is to increase physical fitness and mental fitness and thereby increase the social participation of the person, thereby decreasing the psychological aspect of disability [30]. Exercise also induces an anti-inflammatory effect in the body and strengthens the body's immune response [33, 34]. This concept will be of vital importance in the management of patients post COVID-19 with COPD and Asthma.

3.2.2.1 Effect of aerobic exercise. Aerobic exercise is regarded as the best means to improve muscle functions and exercise tolerance in patients suffering from respiratory diseases [35]. Aerobic exercise could produce short-term safe improvements in the process of immune and respiratory systems. Exercise has an anti-inflammatory role and could improve immunity by improving immune cells and immunoglobulins' function, regulating CRP levels, decreasing anxiety and depression [34]. The exercise also improves respiratory system functions by acting as an antibiotic and antioxidant effect [16]. Supported and unsupported exercises for both upper and lower limbs will be of vital importance using a subjective scale of rating to modulate the intensity of the exercise regime [34].

Patients with COPD and Asthma also show greater exercise intolerance, frequent bouts of dyspnea, more significant participation restrictions, and lower quality of life [36]. Aerobic exercises combined with bronchodilators can remove the struggle with most of the patients initiating the exercise program. The current guidelines for $\mathrm{PR}$ are programmed to two to five times a week for 6-12 weeks $[23,36]$. It's advisable to incorporate a short-acting b2-agonist before $15 \mathrm{~min}$ of starting the exercise to prevent exercise-induced airway hyper-responsiveness [23].

The exercise training workload is categorized based on a cardiopulmonary exercise test or field walking test (60-70\% VO2 max or 60-80-100\% Heart Rate (HR) max) with continuous or interval type of exercise for at least 20-30 min [23]. Mechanism related to the adaptations induced by aerobic exercise remains somewhat hypothetical [23]. It is usually postulated that peripheral muscle adaptations and sensitization due to exercise help reduce feelings of dyspnea, reduce respiratory rate, work of breathing, and bring about positive psychological effects to increase participation at societal levels [23].

Recently it has also been hypothesized that a moderateintensity exercise regime can also play a vital role in modulating the pre-existing inflammatory states, boost the immune response, thereby may prevent or lower down the fatality rates among moderate to severe staging of COPD and Asthma in the population $[17,33]$.

3.2.2.2 Strengthening exercises. This training is an essential aspect of the PR program, which involves both upper and lower limb muscles. The concept is to repetitively lift moderate to high loads to strengthen peripheral muscles, which in turn augments the functional capacity of a person [37]. Another idea is to strengthen respiratory muscles. The approaches of respiratory muscle training (RMT) generally conceptualize on inspiratory muscle training (IMT) and expiratory muscle training (EMT) as training components. Inspiratory muscle training is still an inclusive part of the program, but EMT is still dubious [38].

3.2.2.3 Skeletal muscle strengthening. It is vital to select an appropriate exercise training strategy, which is to provide cardiovascular, pulmonary, and peripheral muscle involvement for the individual patient and should be targeted to maximize the effect of exercise conditioning $[39,40]$. Resistance training of peripheral muscles may be well tolerated and results in a reduction in symptoms of COPD and asthma patients, thereby improving health-related quality of Life [37]. The upper and lower extremities training is designed according to the previous 1 Repetition Maximum (RM) Strength test [23]. The participants usually start with eight repetitions and progress it up to 10 reps. If the exercises are well tolerated, then the load should be increased by $2-10 \%$ [23]. The frequency of training ranges from three to five sessions weekly for a total duration of 15-20 minutes ranging from 6-12 weeks [23]. The Probable advantage of resistance exercise, which involves single muscle groups, may result in lower oxygen consumption and minute ventilation, and therefore it provides additive benefit to the training [23]. 
In the COVID19 era, these training have to be modified to shorter sessions with a careful watch on the patients' oxygen saturation levels $\left(\mathrm{SpO}_{2}\right)$. The affected population may present with profound hypoxemia at rest, without any proportionate signs of respiratory distress (i.e., happy hypoxemia) with rapid worsening [41, 42]. There might be signs of well-preserved oxygen saturation despite low partial pressure of oxygen in arterial blood samples due to the leftward shift of the oxyhemoglobin dissociation curve brought by hypoxemia-driven hyperventilation may possibly result due to direct viral interactions with hemoglobin [43].

3.2.2.4 Respiratory muscle strengthening. There is compelling evidence to support that inspiratory muscle strengthening has ameliorative effects on dyspnea in COPD and is endorsed activities in the pulmonary rehabilitation plan of care [38]. It has been reinstated through metanalysis of randomized controlled trials that IMT improves muscle strength, endurance, functional exercise capacity, reduces symptoms for dyspnea, and enhances the quality of living [44] in COPD. It has also been observed that an eight-week home exercise program with partial supervision to perform inspiratory muscle training (IMT) in patients with COPD and low maximal inspiratory pressure $\left(\mathrm{Pi}_{\max }\right)$ was found to improve respiratory muscle strength and endurance dyspnea, and exercise performance. Dyspnea relief is also reported in trials combined with a lower stimulation of the diaphragm in the absence of any substantial variations in ventilation and breathing pattern [45].

For expiratory muscle training, a randomized controlled trial in COPD conditions outlines that the intervention for five weeks had improvement in the exercise capacity, symptoms, and quality of life. Still, the lung functions remained unchanged post-training [46]. The effect observed may be partially attributed to a change in expiratory muscle strength [46].

Most of the moderate to severe cases in COVID-19 usually affect the lung compliance system causing increased work of breathing and feeling of dyspnea [47]. Requirement of mechanical ventilation with intubation may be regarded as surrogate markers for severe illness and low lung compliance [47]. In such a scenario, post COVID-19 recovery patient during respiratory muscle training (RMT) exercising with a duration of 4 to 8 weeks in hypoxia usually results in (1) decreased respiratory muscle fatigue, (2) late respiratory muscle metaboreflex activation, (3) and better maintenance of oxygen saturation $\left(\mathrm{SaO}_{2}\right)$ and blood flow to the active muscles [48].

\subsubsection{Use of devices for rehabilitation, and precautions during pandemic time}

In PR program, there is usually a need for devices to clear the mucus and improve pulmonary lung functions. These devices are an essential supplement to the program and increase patient adherence as they can use the therapy as a home pro- gram. These devices include the positive expiratory pressure (PEP), the high-frequency chest wall oscillation (HFCWO), the oral high-frequency oscillation, the intrapulmonary percussive ventilation, the incentive spirometry the flutter, and the Acapella and the Cornet. These devices may be administered after therapist discretion [49]. The apparatus, as mentioned earlier, seems to operate in bronchial hygiene and improve airway permeability, $\mathrm{SpO}_{2}$, and dyspnea.

A study found that airway clearance techniques are the most integral part of managing any acute exacerbation in chronic respiratory disease [50]. The study also found that the most frequently used ACTs were PEP devices (90\%), at second directed huffing (88\%), and third cough (71\%) among physical therapy practices for airway management [50].

Usually, PR program is not recommended in the acute stage but only in the latent stage. Still, a noticeable precaution is required to use these devices for lung clearance in the latent stage. As these techniques may generate aerosol, if the patient has a risk of infection or re-infection, using these devices may be limited or done in isolation with appropriate use of personal protective equipment (PPE) is recommended [12]. There is also a need for a separate individualized approach for COPD and asthma patients requiring supplemental devices for treatment.

\subsubsection{Breathing retraining, airways clearance technique and nutritional counselling}

Breathing retraining may be considered as a complementary exercise training that is specifically used to induce relaxation, modulate the high respiratory rate and anxiety which may be encountered in COVID-19 patients with pre-existing COPD and Asthma through its role in pulmonary rehabilitation is still unclear [23]. Studies report that up to $30 \%$ of patients practice breathing techniques to regulate their symptoms [51]. The basic technique for breathing exercise utilizes breathing through the nose with slow breathing and pursedlip expiration which is usually passive; it can be intermixed with controlled breath holds, and relaxation exercises can be managed in person by a therapist or home exercise telerehabilitation [23].

Airway clearance technique (ACT) in PR aims to remove sputum from the lungs during acute exacerbations of COPD or stable COPD [52]. Airway clearance techniques are the most prescribed and trusted techniques used during any acute exacerbations [53]. Authors observed in the survey that most prescribed ACTs among physical therapists were physical exercises (89\%), followed by forced expiratory techniques (81\%) and active cycle of breathing technique (79\%) [53].

Obesity is an important risk factor for COVID-19 and may cause significant morbidity and mortality in the population with obesity [54]. It has been estimated that overweight people are at a higher risk of developing Asthma than patients with normal weights. Obesity is commonly stated comorbidity in asthma patients. It is also reported that the obese population grows a challenging situation to control asthma symp- 
toms, with a poor prognosis of respiratory symptoms and decreased response to corticosteroid therapy [23]. Hence nutritional therapy will play a vital role in controlling obesity and promoting diet with anti-inflammatory effects and enhanced immune response. The western diets that promote obesity tend to be high in saturated fatty acids, sugars, and low fiber and anti-oxidants. Hence a single meal loaded with the aforementioned diet will increase neutrophilic airway inflammation and decreases bronchodilator responsiveness [55].

Moreover, exercise and weight loss programs can also play a decisive role in the program's success for the afflicted population during COVID-19 era.

\subsubsection{Patient self-management of long-term respiratory complications in post COVID-19 population}

Self-management strategies are essential to modify the health-related domain by acquiring the knowledge and skills for self-care in COPD and Asthma [56]. A structured selfmanagement program can reduce the number of hospitalization, thereby reducing the person's social, mental, and physical aspects [56]. A structured self-management program consists of additional education regarding the disease process and respiratory management during acute exacerbations [56]. These self-management strategies can be directly linked to improved quality of life (QoL) in COPD and asthma population [57]. Some studies have also identified investment in leisure time activity also to be directly associated with QoL [57]. Decreased self-management of breathing issues has been associated with a lower level of QoL [57].

\subsubsection{Management of fatigue}

Chronic fatigue syndrome(CFS) is usually reported in rheumatic and other diseases [18]. The prevalence of chronic fatigue in the general population is reported to be $30 \%$. Fatigue-like symptoms may develop in the acute stage of COVID-19 and progresses further as severe chronic fatigue syndrome [18]. As the current situation for COVID-19 is still evolving, early detection and management of post-viral fatigue (PVF) is recommended. A study surveyed participants with symptoms lasting more than 90 days and found the presence of COVID-19 post-viral fatigue [58]. Psychological, social, cognitive, behavioral, and emotional factors may be vital for long-term fatigue [59]. Currently, the nonpharmacological management of PVF in COVID-19 recommends using rehabilitation according to the available evidence $[58,60]$. It is assumed that post-viral fatigue, which may be considered as a subacute form of chronic fatigue syndrome, may have management strategies similar to CFS [59].

It is usually recommended that a graded exercise regime be followed with periodic assessment after recovery [61]. Some authors also suggest that exercise in this population with CFS symptoms should be commenced vigilantly and abstain if the patient develops fever, breathlessness, severe fatigue, or muscle aches [18]. Cognitive therapy, energy conservation techniques, pacing could be vital therapies of management [59].

\subsubsection{Importance of energy conservation techniques}

COVID-19 primarily affects the respiratory system in moderate, moderately severe, and critical cases [62]. Fatigue, anxiety, and depression are also commonly associated with the post-recovery phase [63]. Hence in the pulmonary rehabilitation phase, energy conservation techniques (ECTs) will play an essential role in breaking the vicious cycle of fatigue, anxiety, and depression among the afflicted population. ECTs are precisely intent at performing the activity of daily living (ADL) with low energy cost. To make the patients more functionally independent, it's essential to conserve energy as much as possible. Energy is spent efficiently utilizing conservation techniques for each ADL, thereby constructing day-to-day activities in a thoughtful way [64].

A great number of human activities involve movement of the arms and legs. Even tasks that appear to be simple can cause higher oxygen uptake and minute ventilation, which may also induce the sensation of dyspnea. In the light of current evidence, it is foremost essential to evaluate the impact such activities have on daily life in a population with chronic obstructive pulmonary disease [65]. It will also be necessary to assess the affected population post-recovery to categorize the need for ECTs. A simple way could be by asking the patient to perform a common ADL. At the same time, the therapist examines the reaction time, execution, and total duration of the act, simultaneously recording the heart rate, respiratory rate, oxygen saturation, and dyspnea (if any) following the NYHA scale [65].

Some simple techniques can be used for ECTs as diaphragmatic breathing, controlling the cycles before, during, and after activity to avoid brief periods of apnea. In addition, respiratory exercises can be associated with upper and lower limb training, using subjective scales to monitor training intensity. It is crucial to commence with light, slow activities that require a reduced amount of energy expenditure, such as personal hygiene activities, followed by activities performed in sitting without upper limb support. It is also essential to modify the home and office environment to enhance the ergonomics or use assistive technologies that suit the patient's need [65].

\subsection{Optimizing the ventilation-perfusion (V/Q) matching}

The V/Q ratio estimates the close relationship between ventilation $(\mathrm{V})$ to perfusion $(\mathrm{Q})$ in the lungs. There is local dissimilarity in the V/Q ratio within the lung. Ventilation usually is better at the base of the lung than at the apex [66]. Irregular distribution of both alveolar ventilation and pulmonary blood flow remained the most significant cause of arterial hypoxemia, with or without hypercapnia, in a stable and exacerbated COPD [67]. Airway clearance techniques, especially in the prone position, upper limb, and lower limb exercises, are postulated to improve ventilationperfusion mismatch and lung compliance [42]. 


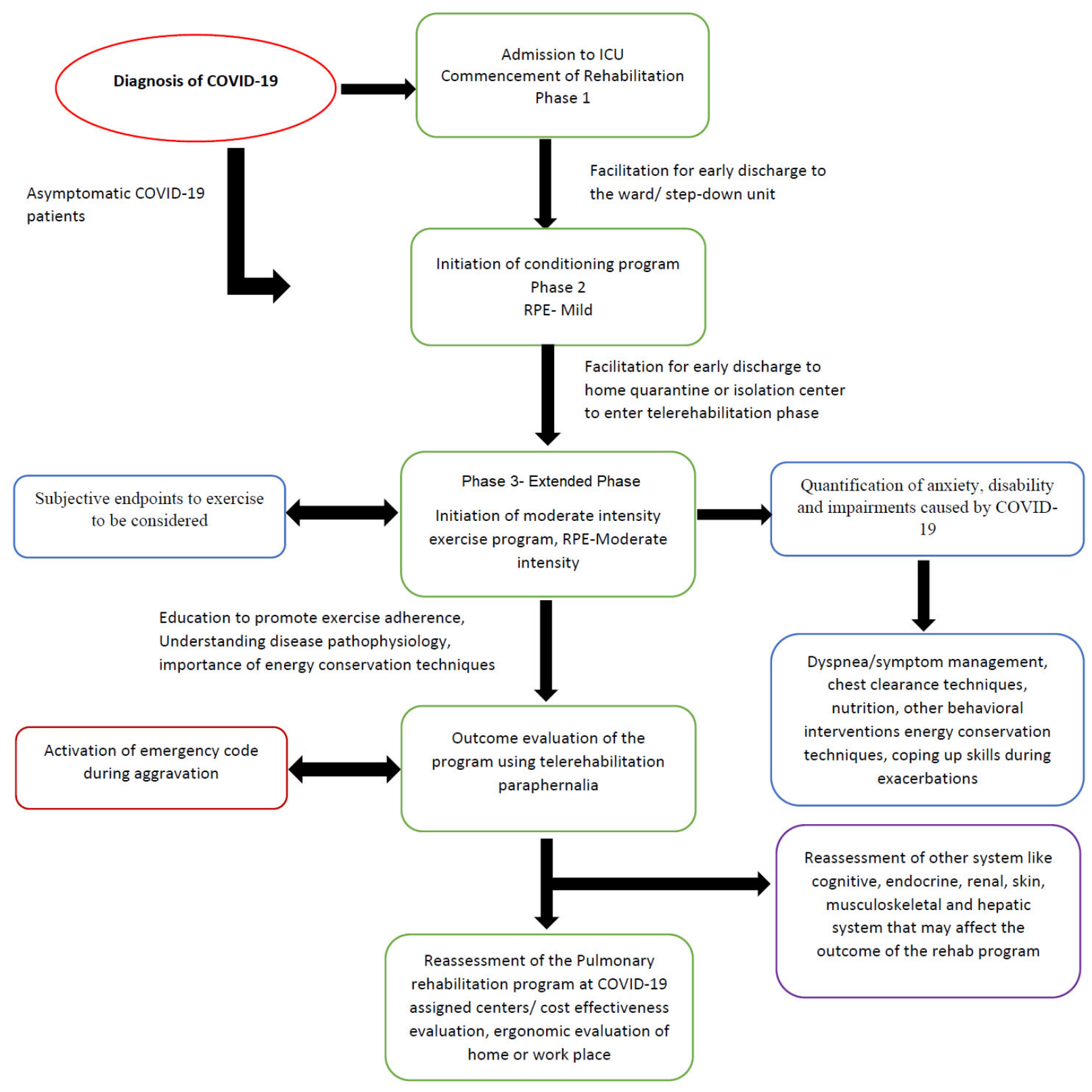

Fig. 1. Proposed flowchart to be followed for the rehabilitation of population afflicted by COVID-19.

\subsection{Robotics in pulmonary rehabilitation}

This is a booming area of research that holds promises for the arena of pulmonary rehabilitation in the future. Pilot studies reinstate robotics to be an effective strategy in facilitating adherence to medications, home-based rehabilitation, enhancing the quality of life, and reducing hospital readmission compared to standard care [68]. Studies have also investigated the role of robotics in PR to recover patients post COVID-19 and its effectiveness in managing the sequelae of prolonged severe illness [69]. Still, the research evidence with robotics is evolving in PR. These innovative technologies will play a vital role in supporting the health care delivery system in the current pandemic scenario.

\section{Need to revise existing PR program}

In the updated scientific brief by World Health Organization (WHO), the organization explicitly states that persons whose symptoms have resolved may still test positive for the COVID-19 virus by reverse transcription-polymerase chain reaction (RT-PCR) for many weeks. But despite these findings, these persons are unlikely to be infectious and therefore are fewer chances to transmit the virus to another person [70]. It is also observed the COVID-19 infection causes multiple organ failure requiring a multidisciplinary approach [42]. 
Table 1. Represents core exercise components to be incorporated in rehabilitation of COVID-19 patients.

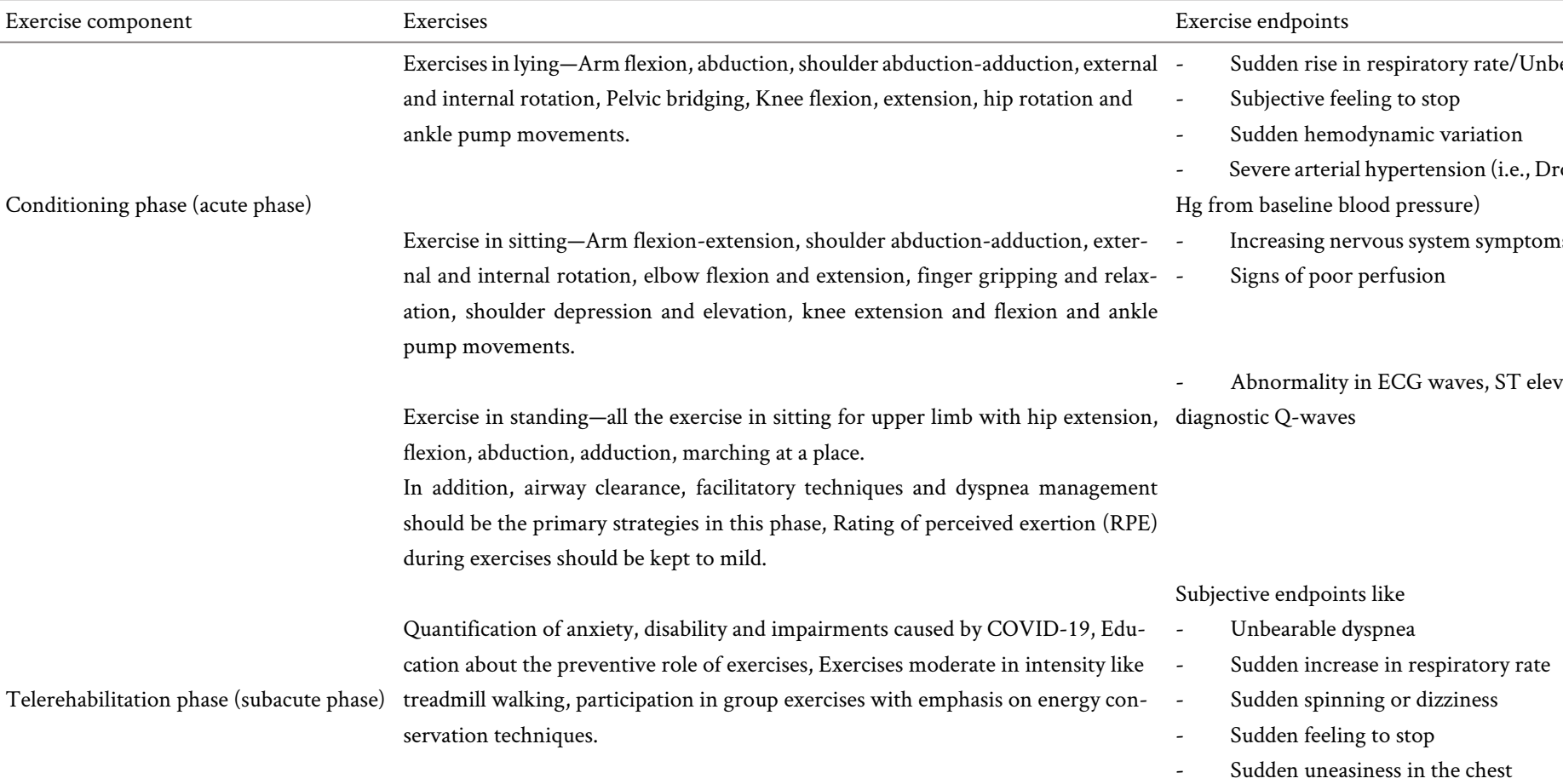

RPE during exercises should be kept to mild-moderate in this phase.

Long term care telerehabilitation phase Reassessing of disability and impairments cause by COVID-19, Ergonomic evalua- Subjective endpoints same as above

tion of the house and workplace, Education for adherence to treatment, nutrition

therapy, Readjustment of exercise plan, activation of the emergency code during aggravation of the problem, maintenance of a diary for recording the exercise frequency and duration daily.

RPE during exercises should be kept to mild-moderate in this phase.

Any transition from phases or within the phase-in exercises must be incorporated with relaxation breathing and coping up strategies like autogenic relaxation, active cycle of breathing techniques with energy conservation techniques. Prone exercises can also be incorporated in lying if vitals are stable as a conditioning exercise. It quite evident now that's its beneficial to the patient population. RPE-Rating of perceived exertion, ECG- Electrocardiogram. 
It is suggestive that important physiotherapy airways management techniques should be incorporated in the pulmonary rehabilitation to improve the quality of life of COVID-19 survivors with moderate to critical cases [71]. A probable flowchart steps that can be followed to counter patients afflicted with COVID-19 (Fig. 1) to be in extended phases of PR to make sure a better follow-up and rehabilitation than the traditional one.

Infection control guidelines: COVID-19 is a highly contagious disease, and it's essential for healthcare professionals not to get contracted themselves with the disease during the rehabilitation process.

ICU rehabilitation: the rehabilitation professionals are the frontrunner team members who usually encounter mildmoderate and severe COVID-19 cases. Addressing the necessity of airway clearance and ventilation-perfusion abnormalities is of utmost need whether the patient is on invasive or noninvasive mechanical ventilation. Mechanical ventilation coupled with prolonged bed rest usually results in respiratory and skeletal muscle dysfunction, early fatigue, breathlessness, delirium, and cognitive changes [72]. It is imperative to initiate the rehabilitation program at the earliest in the disease's latent phase to restore optimum health of the afflicted patients [72]. The incubation period of the COVID-19 is almost five days and recommended isolation period due to infection is 14 days [73].

Pulmonary rehabilitation program: the rehabilitation should start with the patients' specific needs as the therapist may encounter patients (1) Asymptomatic infected patients. (2) Symptomatic patients isolating at home. (3) Symptomatic patients admitted to hospital 4-symptomatic patients requiring ventilatory support as a varying degree of affliction in the population. The above-mentioned situation may be complicated further by the presence of comorbidities like noncommunicable diseases.

There is a greater probability that the patients with COVID-19 with lower immunity may seek readmissions. Hence there is also essential to promote an exercise program that improves immune defense response in such patients once the disease has become latent.

Moderate-intensity exercise and Rehabilitation: there is ample evidence that exercise promotes immunity among healthy individuals [34, 74]. There are some limiting factors in post-COVID-19 survivors to moderate-intensity exercise as symptoms of frequent breathlessness, restrictive type lung disease. Hence before a moderate-intensity exercise program, the person will be required to undergo a conditioning program for 4 weeks (Table 1). In Table 2 uses and clinical implications of various exercises have been outlined in detail.

Components of Exercise rehabilitation: practicing norms of infection control through social distancing is the primary priority. Techniques comprise Proprioceptive Neuromuscular Facilitation techniques (PNF), supported and unsupported arm and leg exercises, breath control techniques, airway clearance techniques, energy conservation techniques, respiratory muscle training to enhance chest expansion and greater gas exchange by recruiting dormant but active alveoli (Tables 1,2). It will be essential to monitor the patient population's oxygen saturation levels to avoid fatigue or aggravate the pre-existing symptoms of breathlessness; hence coping up techniques like energy conservation techniques will play a vital role in the rehabilitation.

Outcome evaluation: it will be essential to incorporate field test like oxygen saturation changes, six-minute walk tests, and step test or shuttle walking test to verify exercise tolerance monitored by $\mathrm{SpO}_{2}$, health-related quality of life questionnaire World Health Organization Quality of Life Instruments (WHOQOL-BREF) [75], is considered an essential outcome of physical exercise training proposed.

National health policy: as it is clear from the current pandemic situation that it will require an endorsement by the national health policymakers also to incorporate the current pulmonary rehabilitation program as part of COVID19 management to ensure continuous availability of the program. And also avoid cessation of the services to the affected population that may seriously compromise the health-related outcomes causing an increase in mortality and morbidity in the population [74].

\section{Scope and future for telerehabilitation}

Telerehabilitation is defined as a telehealth application using telecommunication technologies to administer the rehabilitation services so that patient receives supervised rehabilitation at home while the rehab specialist is at the hospital [76]. It is well established that telerehabilitation used in PR of chronic respiratory diseases produces similar results as compared to center-based PR with no identified safety issues [77]. It is already proven that physiotherapists are uniquely positioned to play an essential role in helping individuals who are at risk of functional decline in the current pandemic situation. Telerehabilitation can play a vital role in monitoring patients post COVID-19 discharge to home or community step-down facility [78].

Currently, the health care services were unprepared for a pandemic of this magnitude [79]. Attracting newer forms of platforms to deliver PR encouraging physical activity in chronic respiratory diseases may be the latest trend in the management [79]. Technological advances like virtual reality, game-based therapy, and telehealth platforms are greatly accepted among the population. They are also an effective strategy in managing chronic respiratory diseases during pandemic times [79]. Hence, remote PR delivery must have a home-based rehabilitation program, virtual rehabilitation and telerehabilitation as an integral component [79].

Tele-rehabilitation in the current pandemic situation will be an extended supportive care utilizing modern paraphernalia to provide inputs for exercise, interaction with the patient for adequate feedback, psychological support, nutritional advice, and apt infection risk management can also be implemented while maintaining isolation and appropriate social distancing [78]. 
Table 2. Effect of Different mode of exercises in respiratory conditions and clinical implications.

\begin{tabular}{lll} 
Mode of exercise training & Exercise-based treatment guidelines and dose parameters & Clinical implication/Training effects \\
\hline & ERS guidelines: &
\end{tabular}

Inspiratory muscle training

Thresto inspiratory muscles

Intensity is set at $30 \%$ to $60 \%$ of the patient's respiratory muscle strength measured as maximum inspiratory pressure.

Daily, one or two times per day with an increment in exercise intensity Weekly is recommended.

ACSM recommendations:

Walking and/or cycling is recommended form of endurance training mode for 3 to 5 days per week with an intensity of

Cardiorespiratory endurance $\quad 30-40 \%$ peak work rate gradually progressing to $60-80 \%$. An intensity of $12-14$ on Borg's Rate of Perceived Exertion training

scale is recommended for the patients.

ATS/ERS recommendations:

Cycling or walking (ground-based or treadmill) is recommended for 3 to 5 times per week with an intensity of more than $60 \%$ of maximal work rate or 12 to 14 on RPE scale and 20 to 60 min of duration per session. The usage of predetermined Metabolic equivalent charts is also used to decide the intensity of exercise.

AACVPR recommendations:

Walking on a treadmill, track, supported walking with the help of a walker, stationary bike exercise, arm ergometry, arm lifting exercises with or without weights, water exercises and swimming which are of light to moderate intensity are recommended for 3-5 times in a week to improve endurance in patients with chronic respiratory conditions.

Unsupported arm training Free weights, lifting a dowel/throwing a ball are the examples for upper limb endurance exercises. The training intensity Improved upper limb endurance and improved activities of at a rate of 15 repetitions with a starting weight approximately for ten minutes, the number of sets gradually increased daily living (mainly ADL activities where upper limbs are to three, and then the intensity is increased by $0.5 \mathrm{~kg}$. Cycle ergometry is recommended for two times per week. 
Table 2. Continued.

\begin{tabular}{|c|c|c|}
\hline Mode of exercise training & Exercise-based treatment guidelines and dose parameters & Clinical implication/Training effects \\
\hline $\begin{array}{l}\text { Peripheral muscle strengthening- } \\
\text { both upper and lower limbs }\end{array}$ & $\begin{array}{l}\text { ACSM recommendations: Free weights, machines with stacked weights or pneumatic resis- } \\
\text { tance, and resistance bands for more than } 2 \text { days per week at an intensity of } 40-70 \% 1 \mathrm{RM} \\
\text { with a gradual progression is recommended. } \\
\text { ATS/ERS recommendations: Resistance exercise for } 2 \text { to } 3 \text { times per week with an initial } \\
\text { intensity of } 40-50 \% 1 \text { Repetition Maximum gradually progressed to } 60-70 \% 1 \mathrm{RM} \text { or } 100 \% \\
\text { of 8-12 RM is recommended. } \\
\text { AACVPR recommendations: The recommendations state that monitoring the rate of per- } \\
\text { ceived exertion and fatigue and pain is required for training. The resistance exercises should } \\
\text { begin with lower resistance and higher repetitions, further progress to higher weights and } \\
\text { fewer repetitions. }\end{array}$ & $\begin{array}{l}\text { Improved muscle strength, improved balance, and health-related } \\
\text { quality of life }\end{array}$ \\
\hline Flexibility training & $\begin{array}{l}\text { ACSM recommendations: Recommends the use of slow movements that involve sustained } \\
\text { stretches for each major muscle group for more than } 2 \text { days per week. }\end{array}$ & Improved flexibility and decreased risk of falls \\
\hline Balance training & $\begin{array}{l}\text { AACVPR recommends static and dynamic stance exercises tandem, sideways and backward } \\
\text { walking, Tai Chi, modified yoga for whole-body stretching with coordinated breathing to } \\
\text { improve balance in patients with chronic pulmonary diseases and suitable for Pulmonary } \\
\text { Rehabilitation. }\end{array}$ & Improved balance and decreased risk of falls \\
\hline
\end{tabular}

MIP, Maximum Inspiratory Pressure; RPE, Rate of Perceived Exertion; MET, Metabolic equivalent; RM, Repetition Maximum; 6MWT, Six-Minute Walk Test; ISWT, Incremental Shuttle Walk test; ACSM, American College of Sports Medicine; AACVPR, American Association of Cardiovascular and Pulmonary Rehabilitation; ERS, European Respiratory Society; ATS, American Thoracic Society. 


\section{Conclusions}

In conclusion, there is an emerging need for healthcare professionals to deal with chronic COPD and asthma patients during the COVID-19 era. The therapist should design an individually tailored PR program to improve the chronic symptoms and reduce complications among the population with respiratory diseases. Emphasis on airway clearance techniques for the management of cough, sputum, and breathlessness will play a vital role in promoting well-being. Energy conservation techniques can be used to encourage the activity of daily living among severe cases. Moreover, telerehabilitation and robotics technology may have a significant role in rehabilitation during the pandemic situation.

\section{Author contributions}

$\mathrm{SD}, \mathrm{ABS}$ and KCB designed the Review study. SD and KCB performed the search. SD and ABS provided help and advices to select and design the draft. SD and ABS analyzed the data. All authors contributed to editorial changes in the manuscript. All authors read and approved the final manuscript.

\section{Ethics approval and consent to participate Not applicable.}

\section{Acknowledgment}

We are thankful to King Khalid University for providing us the access to the required documents and studies relevant to the research.

\section{Funding}

This research received no external funding.

\section{Conflict of interest}

The authors declare no conflict of interest.

\section{References}

[1] Leung JM, Yang CX, Tam A, Shaipanich T, Hackett T, Singhera GK, et al. ACE-2 expression in the small airway epithelia of smokers and COPD patients: implications for COVID-19. European Respiratory Journal. 2020; 55: 2000688.

[2] Lippi G, Henry BM. Chronic obstructive pulmonary disease is associated with severe coronavirus disease 2019 (COVID-19): COPD and COVID-19. Respiratory Medicine. 2020; 16.

[3] Blumenthal D, Fowler EJ, Abrams M, Collins SR. COVID-19implications for the health care system. New England Journal of Medicine. 2020; 383: 1483-1488.

[4] Zayet S, Kadiane-Oussou NJ, Lepiller Q, Zahra H, Royer P, Toko $\mathrm{L}$, et al. Clinical features of COVID-19 and influenza: a comparative study on Nord Franche-Comte cluster. Microbes and Infection. 2020; 22: 481-488.

[5] Rothan HA, Byrareddy SN. The epidemiology and pathogenesis of coronavirus disease (COVID-19) outbreak. Journal of Autoimmunity. 2020; 109: 102433.

[6] Engelkes M, de Ridder MA, Svensson E, Berencsi K, PrietoAlhambra D, Lapi F, et al. Multinational cohort study of mortality in patients with asthma and severe asthma. Respiratory Medicine. 2020; 165: 105919.

[7] Wu F, Zhou Y, Wang Z, Xie M, Shi Z, Tang Z, et al. Clinical characteristics of COVID-19 infection in chronic obstructive pulmonary disease: a multicenter, retrospective, observational study. Journal of Thoracic Disease. 2020; 12: 1811-1823.

[8] 2021. Available at: https://goldcopd.org/wp-content/uploads /2020/11/GOLD-REPORT-2021-v1.1-25Nov20_WMV.pdf (Accessed: 4 April 2021).

[9] Chang MC, Park Y, Kim B, Park D. Risk factors for disease progression in COVID-19 patients. BMC Infectious Diseases. 2020; 20: 445.

[10] Grasselli G, Greco M, Zanella A, Albano G, Antonelli M, Bellani G, et al. Risk factors associated with mortality among patients with COVID-19 in intensive care units in Lombardy, Italy. JAMA Internal Medicine. 2020; 108: 1345-1355.

[11] Celli B, Fabbri LM. Urgent need of a management plan for survivors of COVID-19. European Respiratory Journal. 2020; 55: 2000764.

[12] Kurtaiş Aytür Y. Pulmonary rehabilitation principles in SARS$\mathrm{CoV}-2$ infection (COVID-19): a guideline for the acute and subacute rehabilitation. Turkish Journal of Physical Medicine and Rehabilitation. 2020; 66: 104-120.

[13] Anastasio F, Barbuto S, Scarnecchia E, Cosma P, Fugagnoli A, Rossi G, et al. Medium-term impact of COVID-19 on pulmonary function, functional capacity and quality of life. European Respiratory Journal. 2021. (in press)

[14] Spagnolo P, Balestro E, Aliberti S, Cocconcelli E, Biondini D, Casa GD, et al. Pulmonary fibrosis secondary to COVID-19: a call to arms? The Lancet Respiratory Medicine. 2020; 8: 750-752.

[15] Satia I, Cusack R, Greene JM, O’Byrne PM, Killian KJ, Johnston N. Prevalence and contribution of respiratory viruses in the community to rates of emergency department visits and hospitalizations with respiratory tract infections, chronic obstructive pulmonary disease and Asthma. PLoS ONE. 2020; 15: e0228544.

[16] Adamopoulos S, Parissis J, Karatzas D, Kroupis C, Georgiadis M, Karavolias G, et al. Physical training modulates proinflammatory cytokines and the soluble Fas/soluble Fas ligand system in patients with chronic heart failure. Journal of the American College of Cardiology. 2002; 39: 653-663.

[17] Wang TJ, Chau B, Lui M, Lam G, Lin N, Humbert S. Physical medicine and rehabilitation and pulmonary rehabilitation for COVID-19. American Journal of Physical Medicine \& Rehabilitation. 2020; 99: 769-774.

[18] Salisbury H. Helen Salisbury: when will we be well again? British Medical Journal. 2020; 369: 32248536.

[19] Zhao Q, Meng M, Kumar R, Wu Y, Huang J, Lian N, et al. The impact of COPD and smoking history on the severity of COVID-19: a systemic review and meta-analysis. Journal of Medical Virology. 2020; 92: 1915-1921.

[20] Grasselli G, Tonetti T, Protti A, Langer T, Girardis M, Bellani G, et al. Pathophysiology of COVID-19-associated acute respiratory distress syndrome: a multicentre prospective observational study. The Lancet Respiratory Medicine. 2020; 8: 1201-1208.

[21] Spruit MA, Vercoulen JH, Sprangers MAG, Wouters EFM. Fatigue in COPD: an important yet ignored symptom. The Lancet. Respiratory Medicine. 2017; 5: 542-544.

[22] Marques A, Jácome C, Rebelo P, Paixão C, Oliveira A, Cruz J, et al. Improving access to community-based pulmonary rehabilitation: $3 \mathrm{R}$ protocol for real-world settings with cost-benefit analysis. BMC Public Health. 2019; 19: 676.

[23] Zampogna E, Zappa M, Spanevello A, Visca D. Pulmonary rehabilitation and asthma. Frontiers in Pharmacology. 2020; 11: 1-6.

[24] Goodman-Casanova JM, Dura-Perez E, Guzman-Parra J, CuestaVargas A, Mayoral-Cleries F. Telehealth home support during COVID-19 confinement for community-dwelling older adults with mild cognitive impairment or mild dementia: survey study. Journal of Medical Internet Research. 2020; 22: e19434.

[25] Dew A, Bulkeley K, Veitch C, Bundy A, Gallego G, Lincoln M, et al. Addressing the barriers to accessing therapy services in rural and remote areas. Disability and Rehabilitation. 2013; 35: $1564-$ 1570 . 
[26] Blackstock FC, Evans RA. Rehabilitation in lung diseases: 'Education' component of pulmonary rehabilitation. Respirology. 2019; 24: 863-870.

[27] Jaywant A, Vanderlind WM, Boas SJ, Dickerman AL. Behavioral interventions in acute COVID-19 recovery: a new opportunity for integrated care. General Hospital Psychiatry. 2020; 69: 113-114.

[28] Askey R. Exploring the benefits of a psychoeducation session for patients with chronic obstructive pulmonary disease (COPD) and co-morbid depression and/or anxiety. Mental Health. 2020.

[29] Aldcroft SA, Taylor NF, Blackstock FC, O'Halloran PD. Psychoeducational rehabilitation for health behavior change in coronary artery disease. Journal of Cardiopulmonary Rehabilitation and Prevention. 2011; 31: 273-281.

[30] Folgering H, van Herwaarden C. Pulmonary rehabilitation in asthma and COPD, physiological basics. Respiratory Medicine. 1993; 87: 41-44.

[31] Strategy G, Obstructive C, Disease P. GOLD (Global Initiative for Chronic Obstructive Lung Disease) 2017. Pneumologie. 2017; 71: 9-14.

[32] Palange P, Ward SA, Carlsen K, Casaburi R, Gallagher CG, Gosselink R, et al. Recommendations on the use of exercise testing in clinical practice. European Respiratory Journal. 2007; 29: 185209.

[33] Pedersen BK, Steensberg A, Schjerling P. Exercise and interleukin6. Current Opinion in Hematology. 2001; 8: 137-141.

[34] Dixit S. Can moderate intensity aerobic exercise be an effective and valuable therapy in preventing and controlling the pandemic of COVID-19? Medical Hypotheses. 2020; 143: 109854.

[35] Spruit MA, Burtin C, De Boever P, Langer D, Vogiatzis I, Wouters EFM, et al. COPD and exercise: does it make a difference? Breathe. 2016; 12: e38-e49.

[36] Bernard S, Ribeiro F, Maltais F, Saey D. Prescribing exercise training in pulmonary rehabilitation: a clinical experience. Revista Portuguesa de Pneumologia. 2014; 20: 92-100.

[37] Lee AL, Holland AE. Time to adapt exercise training regimens in pulmonary rehabilitation-a review of the literature. International Journal of Chronic Obstructive Pulmonary Disease. 2014; 9: 1275-1288.

[38] Garcia S, Rocha M, Pinto P, M F Lopes A, Bárbara C. Inspiratory muscle training in COPD patients. Revista Portuguesa de Pneumologia. 2008; 14: 177-194.

[39] Daabis R, Hassan M, Zidan M. Endurance and strength training in pulmonary rehabilitation for COPD patients. Egyptian Journal of Chest Diseases and Tuberculosis. 2017; 66: 231-236.

[40] Vogiatzis I. Strategies of muscle training in very severe COPD patients. The European Respiratory Journal. 2011; 38: 971-975.

[41] Dechman G, Aceron R, Beauchamp M, Bhutani M, Bourbeau J, Brooks D, et al. Delivering pulmonary rehabilitation during the COVID-19 pandemic: a Canadian Thoracic Society position statement. Canadian Journal of Respiratory, Critical Care, and Sleep Medicine. 2020; 4: 232-235.

[42] Gautam AP, Arena R, Dixit S, Borghi-Silva A. Pulmonary rehabilitation in COVID-19 pandemic era: The need for a revised approach. 2020. Available at: https://onlinelibrary.wiley.com/doi/ 10.1111/resp.13946 (Accessed: 3 April 2021).

[43] Dhont S, Derom E, Van Braeckel E, Depuydt P, Lambrecht BN. The pathophysiology of 'happy' hypoxemia in COVID-19. Respiratory Research. 2020; 21: 198.

[44] Gosselink R, De Vos J, van den Heuvel SP, Segers J, Decramer M, Kwakkel G. Impact of inspiratory muscle training in patients with COPD: what is the evidence? The European Respiratory Journal. 2011; 37: 416-425.

[45] Langer D, Ciavaglia C, Faisal A, Webb KA, Neder JA, Gosselink R, et al. Inspiratory muscle training reduces diaphragm activation and dyspnea during exercise in COPD. Journal of Applied Physiology. 2018; 125: 381-392.

[46] Mota S, Güell R, Barreiro E, Solanes I, Ramírez-Sarmiento A, Orozco-Levi $\mathrm{M}$, et al. Clinical outcomes of expiratory muscle training in severe COPD patients. Respiratory Medicine. 2007;
101: 516-524.

[47] Komorowski M, Aberegg SK. Using applied lung physiology to understand COVID-19 patterns. British Journal of Anaesthesia. 2020; 125: 250-253.

[48] Álvarez-Herms J, Julià-Sánchez S, Corbi F, Odriozola-Martínez A, Burtscher M. Putative role of respiratory muscle training to improve endurance performance in hypoxia: a review. Frontiers in Physiology. 2019; 9: 1970.

[49] Hristara-Papadopoulou A, Tsanakas J, Diomou G, Papadopoulou O. Current devices of respiratory physiotherapy. Hippokratia. 2008; 12: 211-220.

[50] Westerdahl E, Osadnik C, Emtner M. Airway clearance techniques for patients with acute exacerbations of chronic obstructive pulmonary disease: Physical therapy practice in Sweden. Chronic Respiratory Disease. 2019; 16: 147997311985586.

[51] Ernst E. Breathing techniques-adjunctive treatment modalities for asthma? A systematic review. The European Respiratory Journal. 2000; 15: 969-972.

[52] Osadnik CR, McDonald CF, Jones AP, Holland AE. Airway clearance techniques for chronic obstructive pulmonary disease. The Cochrane Database of Systematic Reviews. 2012; CD008328.

[53] Osadnik CR, McDonald CF, Holland AE. Airway clearance techniques in acute exacerbations of COPD: a survey of Australian physiotherapy practice. Physiotherapy. 2013; 99: 101-106.

[54] Popkin BM, Du S, Green WD, Beck MA, Algaith T, Herbst CH, et al. Individuals with obesity and COVID-19: a global perspective on the epidemiology and biological relationships. Obesity Reviews. 2020; 21: e13128.

[55] O’Neill K, O’Donnell AE, Bradley JM. Airway clearance, mucoactive therapies and pulmonary rehabilitation in bronchiectasis. Respirology. 2019; 24: 227-237.

[56] Yu S, Guo A, Zhang X. Effects of self-management education on quality of life of patients with chronic obstructive pulmonary disease. International Journal of Nursing Sciences. 2014; 1: 53-57.

[57] Newham JJ, Presseau J, Heslop-Marshall K, Russell S, Ogunbayo OJ, Netts P, et al. Features of self-management interventions for people with COPD associated with improved health-related quality of life and reduced emergency department visits a systematic review and meta-analysis. International Journal of Chronic Obstructive Pulmonary Disease. 2017; 12: 1705-1720.

[58] Lapp CW, John JF. Managing COVID-19 post viral fatigue syndrome. Fatigue: Biomedicine, Health \& Behavior. 2021; 9: 1-8.

[59] Gaber T. Assessment and management of post-COVID fatigue. Progress in Neurology and Psychiatry. 2021; 25: 36-39.

[60] Barker-Davies RM, O'Sullivan O, Senaratne KPP, Baker P, Cranley M, Dharm-Datta S, et al. The Stanford Hall consensus statement for post-COVID-19 rehabilitation. British Journal of Sports Medicine. 2020; 54: 949-959.

[61] Greenhalgh T, Knight M, A'Court C, Buxton M, Husain L. Management of post-acute COVID-19 in primary care. British Medical Journal. 2020; 370.

[62] Tang X, Du R, Wang R, Cao T, Guan L, Yang C, et al. Comparison of hospitalized patients with ARDS caused by COVID-19 and H1N1. Chest. 2020; 158: 195-205.

[63] Islam MF, Cotler J, Jason LA. Post-viral fatigue and COVID-19: lessons from past epidemics. Fatigue: Biomedicine, Health \& Behavior. 2020; 8: 61-69.

[64] Wingårdh A, Göransson C, Larsson S, Slinde F, Vanfleteren LGW. Effectiveness of energy conservation techniques in patients with COPD. Respiration. 2020; 99: 409-416.

[65] Velloso M, Jardim JR. Functionality of patients with chronic obstructive pulmonary disease: energy conservation techniques. Jornal Brasileiro de Pneumologia. 2006; 32: 580-586.

[66] Wagner PD. Ventilation-perfusion matching during exercise. Chest. 1992; 101: 192S-198S.

[67] Rodríguez-Roisin R, Drakulovic M, Rodríguez DA, Roca J, Barberà JA, Wagner PD. Ventilation-perfusion imbalance and chronic obstructive pulmonary disease staging severity. Journal of Applied Physiology. 2009; 106: 1902-1908. 
[68] Broadbent E, Garrett J, Jepsen N, Li Ogilvie V, Ahn HS, Robinson $\mathrm{H}$, et al. Using robots at home to support patients with chronic obstructive pulmonary disease: pilot randomized controlled trial. Journal of Medical Internet Research. 2018; 20: e45.

[69] Tay SS, Neo EJ, Tan MM, Tan PL. Post-Critical Care COVID-19 Patient benefits from a robotic patient-guided suspension system for pulmonary rehabilitation. Annals of the Academy of Medicine, Singapore. 2020; 49: 401-404.

[70] States M. Criteria for releasing COVID-19 patients from isolation. World Health Organization. 2020; 1-5.

[71] Thomas P, Baldwin C, Bissett B, Boden I, Gosselink R, Granger $\mathrm{CL}$, et al. Physiotherapy management for COVID-19 in the acute hospital setting: Recommendations to guide clinical practice. Pneumonia. 2020; 33: 32-35.

[72] PAHO-WHO. Rehabilitation considerations during the outbreak COVID-19. 2020. Available at: www.paho.org/coronavirus (Accessed: 1 April 2021).

[73] . WHO. Coronavirus disease 2019 (COVID-19) Situation Report - 73. Available at: https://www.who.int/docs/default-source/co ronaviruse/situation-reports/20200402-sitrep-73-covid-19.pdf? sfvrsn=5ae25bc7_6 (Accessed: 2 April 2021).

[74] Dimitrov S, Hulteng E, Hong S. Inflammation and exercise: inhi- bition of monocytic intracellular TNF production by acute exercise via $\beta 2$-adrenergic activation. Brain, Behavior, and Immunity. 2016; 61: 60-68.

[75] Algahtani FD, Hassan SUN, Alsaif B, Zrieq R. Assessment of the quality of life during COVID-19 pandemic: A cross-sectional survey from the kingdom of Saudi Arabia. International Journal of Environmental Research and Public Health. 2021; 18: 1-12.

[76] Paneroni M, Colombo F, Papalia A, Colitta A, Borghi G, Saleri $\mathrm{M}$, et al. Is telerehabilitation a safe and viable option for patients with COPD? A Feasibility Study. Chronic Obstructive Pulmonary Disease. 2015; 12: 217-225.

[77] Cox NS, Mcdonald CF, Hill CJ, O'Halloran P, Alison JA, Zanaboni $\mathrm{P}$, et al. Telerehabilitation for chronic respiratory disease. Cochrane Database of Systematic Reviews. 2018; 2018: CD013040.

[78] Salawu A, Green A, Crooks MG, Brixey N, Ross DH, Sivan M. A proposal for multidisciplinary tele-rehabilitation in the assessment and rehabilitation of COVID-19 survivors. International Journal of Environmental Research and Public Health. 2020; 17: 1-13.

[79] Rutkowski S. Management challenges in chronic obstructive pulmonary disease in the COVID-19 pandemic: telehealth and virtual reality. Journal of Clinical Medicine. 2021; 10: 1261. 